\title{
Beckert, Sven/Sachsenmaier, Dominic (Hrsg.): Global History, Globally. Research and Practice around the World, 320 S., Bloomsbury, London/New York 2018.
}

\author{
Martin Aust
}

Online publiziert: 25. März 2020

(C) Der/die Autor(en) 2020

Die Geschichtswissenschaft hat im zurückliegenden Vierteljahrhundert eine ganze Reihe von Umbrüchen erfahren. Der jüngste und das Attribut turn vollkommen verdienende ist dabei der Umbruch zur Globalgeschichte gewesen. Eine gerade in den letzten Jahren zunehmende Zahl von Studiengängen, Zentren, Konferenzen und Buchpublikationen führt die Bezeichnung Globalgeschichte in ihrem Titel. Globalgeschichte setzt sich mit dem Erbe der Geschichtswissenschaft des 19. und 20. Jahrhunderts kritisch auseinander: Nationale Infrastrukturen und Paradigmata standen an der Wiege universitärer Geschichtsschreibung in der Mitte des 19. Jahrhunderts. Der Geschichtsschreibung in Europa lag eine eurozentrische Weltsicht zugrunde. Dieses Erbe hat die Globalgeschichte aufgegriffen und dabei Nationen, Regionen und die Dominanz des Westens im 19. und 20. Jahrhundert in globalen Kontexten historisiert. Die Globalgeschichte orientiert sich an Methodiken der Verflechtungsgeschichte und des Vergleichs. Sie fragt nach lokalen Ausprägungen von Globalität. In Abgrenzung von der Kulturgeschichte der 1990er Jahre hat die Globalgeschichte Kausalität wieder auf die Agenda der Geschichtsschreibung gesetzt und blickt dabei insbesondere auf die Geschichten von Umwelt, Demografie und Ökonomie. Zugleich ist die Globalgeschichte selbst ein Möglichkeitsraum der vernetzten Welt, in der auch Historiker globale und grenzüberschreitende Erfahrungen in ihrer Arbeit machen. Wer Globalgeschichte studieren möchte, findet entsprechend Studiengänge und Zentren in Beijing, Shanghai, Osaka, São Paulo, Dakar, Oxford und Berlin, um nur einige Orte zu nennen. Sie zeigen, dass die Globalgeschichtsschreibung selbst global geworden ist.

Dies nehmen Sven Beckert und Dominic Sachsenmaier zum Anlass, eine Zwischenbilanz des Feldes vorzulegen. Sie fragen danach, wie Globalgeschichte in un-

M. Aust $(\bowtie)$

Rheinische Friedrich-Wilhelms-Universität Bonn, Bonn, Deutschland

E-Mail: martin.aust@uni-bonn.de 
terschiedlichen Weltregionen und Ländern aufgefasst wird und welche Perspektiven das für die Globalgeschichtsschreibung eröffnet. Konzeption und Praxis der Globalgeschichte weltweit rückt der Band in den Mittelpunkt. Auf eine konzise Einleitung folgen drei große Themenblöcke, die Regionen, zentralen Themen und praktischen Herausforderungen der Globalgeschichte gewidmet sind. In ihrer Einleitung konzedieren Beckert und Sachsenmaier, dass lokale Bedingungen die Auffassung von Globalität in der Geschichtswissenschaft konditionieren. Umgekehrt beeinflussen globale Trends lokale und regionale Historiografien. Beckert und Sachsenmaier ist unbedingt zuzustimmen, wenn sie festhalten, dass Globalgeschichte allein als weltumspannendes, transkontinentales Gespräch funktionieren kann. Sie kritisieren eine nach wie vor zu beobachtende Asymmetrie in der Rezeption von Historiografien: Während es unter Globalhistorikern in Asien eine Selbstverständlichkeit sei, Forschungen aus Europa und den USA zu rezipieren, gelte dies in umgekehrter Richtung nicht. Gerade dies konfrontiere das Projekt der Globalgeschichte mit einer fundamentalen Herausforderung: „How powerful can historians' arguments be, if the field's practicioners in the West continue to ignore scholarship produced in other parts of the world?" fragen Beckert und Sachsenmaier in ihrer Einleitung (S. 9).

Regional unterschiedliche Traditionen und Perspektiven von Globalgeschichte erhellt der erste Teil des Bandes. Gareth Austin bilanziert den Stand der Globalgeschichte in Westeuropa und hält fest, dass der „Eurocentrism of agency“ überwunden sei, ein ,conceptual Eurocentrism“ insgesamt jedoch noch feststellbar sei. Die Geschichtsschreibungen im westlichen Europa geben eine Momentaufnahme zwischen Vernetzung in der Welt und einem konzeptionellen Traditionsüberhang der Fokussierung auf Europa ab. Q. Edward Wang konstatiert ähnliche historiografische Trends seit der Mitte des 19. Jahrhunderts in Korea, Japan und China. Dies gilt auch für das Aufkommen der Globalgeschichte in den zurückliegenden Jahrzehnten. Die Geschichtsschreibung in allen drei Ländern zeigt sich offen für relationale Geschichten. Zugleich haben aber auch nationale Perspektiven, nicht zuletzt in der Globalgeschichte, sich als hartleibig in allen drei Ländern erwiesen. Rafael Marquese und João Paulo Pimenta arbeiten heraus, dass die Historiografien in Lateinamerika über die gesamten zurückliegenden 150 Jahre sensibel für transregionale und transkontinentale Verflechtungen gewesen sind. Der Zusammenhang zwischen der karibischen Sklaverei und dem ökonomischen Aufstieg Europas, der Austausch zwischen Fernand Braudel und brasilianischen Historikern über den Kapitalismus als Weltsystem und schließlich Dependency Studies von lateinamerikanischen Ökonomen und Soziologen sind drei Beispiele für globale Perspektiven über die Jahrzehnte in den Historiografien Lateinamerikas. Omar Gueye unterstreicht, dass eine Geschichte Afrikas in der Neuzeit undenkbar wäre ohne die Berücksichtigung transkontinentaler Verknüpfungen. Gleichzeitig hat aber auch die Zurückweisung des europäischen Stereotyps von Afrika als einem Kontinent ohne Geschichte dazu geführt, dass die Staatsbildungsprozesse in Afrika im 20. Jahrhundert von ausgesprochen nationalen Geschichten begleitet wurden. Die Beschreibung des Eigenen in Auseinandersetzung mit der europäischen Fremdbeschreibung erfolgte im Zeichen des Nationalen. Der jüngste Trend in den Historiografien Afrikas weise jedoch sehr stark in Richtung einer Verknüpfung des Lokalen mit dem Globalen. Selçuk Esenbel und Meltem Toksöz bestätigen dem späten Osmanischen Reich eine vielfältige, differenzierte 
Historiografie. In ihr hatte sich auch eine Weltgeschichtsschreibung etabliert, die sich paradigmatisch von westlicher Geschichtsschreibung unterschied. Auf das Ende des Osmanischen Reiches folgten sowohl in der Türkei als auch in den arabischen Nachfolgestaaten des Reiches Nationalhistoriografien. Während des Kalten Krieges gab es in der türkischen Historiografie eine Debatte, in der sich Vertreter modernisierungstheoretischer und weltsystemischer Ansätze gegenüberstanden. Seit den 1980er Jahren hat sich auch die Historiografie in der Türkei Positionen der alternativen und geteilten Moderne zu eigen gemacht. Eine Renaissance der Beschäftigung mit dem Osmanischen Reich und die transnationale Mobilität von Historikern im Nahen Osten haben jüngst auch transnationale Ansätze stark werden lassen. Jerry $\mathrm{H}$. Bentley sieht keinen kategorialen Unterschied zwischen Weltgeschichte und Globalgeschichte. Die Weltgeschichte habe in den USA schon seit den 1960er Jahren und damit lange vor dem Aufkommen der Globalgeschichte daran gearbeitet, nationale Rahmungen und den Eurozentrismus infrage zu stellen. Der jüngste Boom der Globalgeschichte sei vor allem von Historikerinnen und Historikern getragen worden, die sich dem liberalen Lager zurechnen ließen. Beobachten lasse sich dies an einer rechten Kritik an Projekten globaler Geschichte in den USA, die den Globalhistorikern vorwirft, nationale Identität zu untergraben. Diese Beiträge im ersten Teil des Sammelbandes zeichnen ein facettenreiches Bild der Ursprünge und aktuellen Stände von Globalgeschichte in allen Weltregionen.

Der zweite Teil des Buches behandelt Themen der Globalgeschichte: Arbeit, Ökonomie, Migration und Intellectual History. Die Beiträge lesen sich mit Gewinn und geben informierte Überblicke über ihr Themengebiet. Gleichwohl scheint dieser Teil des Sammelbandes etwas kurz geraten. Eine Reihe von weiteren Themen dürfen einschlägig für die Globalgeschichte gelten, die in diesem Band nicht präsent sind: Infrastrukturen der Kommunikation und Imperiengeschichte etwa. Der Band klingt in seinem dritten Teil mit Beiträgen zu Herausforderungen in der Globalgeschichte aus. Eine große Problematik ist das Verhältnis von Globalgeschichte und Nationalgeschichte geblieben. In einigen Kontexten erfährt Globalgeschichte eine Kritik im Namen der Nation. In anderen Kontexten kann eine globalgeschichtliche Perspektive wiederum das Thema Nation umso stärker auf die Agenda setzen. Die Gestalt des Verhältnisses zwischen Globalgeschichte und Nation variiert lokal. David Simo fragt, wie afrikanische Historiker eine Stimme Afrikas in den Diskurs der Globalgeschichte einbringen können. Er plädiert für eine bewusste Auseinandersetzung mit den Themen und Paradigmata der Globalgeschichte aus dem globalen Norden. Erst Präsenz und Aktivität von Historikerinnen und Historikern aus dem globalen Süden im globalen Norden wird Afrika einen Platz im Projekt der Globalgeschichte sichern können. Lim Jie-Hyun zeigt am Beispiel Japans und Koreas, wie nationale Paradigmata in einer patriotischen Geschichtsauffassung und transnationale Geschichtsprojekte miteinander in Konkurrenz stehen. Zwischen den nationalen Geschichtsprojekten in Ostasien gebe es eine antagonistische Komplizenschaft gegenüber der Globalgeschichte. Marnie Hughes-Warrington erhellt Perspektiven der Globalgeschichte in Australien als postkolonialer Siedlergesellschaft. Globalgeschichte als Thema in Forschung und Lehre sei in Australien parallel zur Befassung mit der Geschichte der Aborigines entstanden. In der Aboriginal History sieht Hughes-Warrington ein großes Vermögen, eine alternative Globalgeschichte 
zu entwickeln. Shigeru Akita hebt schließlich das große Potenzial hervor, welchen Beitrag die japanische Wirtschaftsgeschichtsschreibung zu einer Überwindung eurozentrischer Positionen in der Wirtschaftsgeschichte leisten kann.

Sven Beckert und Dominic Sachsenmaier haben einen perspektivenreichen Sammelband vorgelegt, der seinen Platz im Lektürekanon der Globalgeschichte finden wird. Der Band überzeugt vor allem mit seinen erhellenden Einblicken in die lokalen Werkstätten der Globalgeschichte auf allen Kontinenten.

Funding Open Access funding provided by Projekt DEAL.

Open Access Dieser Artikel wird unter der Creative Commons Namensnennung 4.0 International Lizenz veröffentlicht, welche die Nutzung, Vervielfältigung, Bearbeitung, Verbreitung und Wiedergabe in jeglichem Medium und Format erlaubt, sofern Sie den/die ursprünglichen Autor(en) und die Quelle ordnungsgemäß nennen, einen Link zur Creative Commons Lizenz beifügen und angeben, ob Änderungen vorgenommen wurden.

Die in diesem Artikel enthaltenen Bilder und sonstiges Drittmaterial unterliegen ebenfalls der genannten Creative Commons Lizenz, sofern sich aus der Abbildungslegende nichts anderes ergibt. Sofern das betreffende Material nicht unter der genannten Creative Commons Lizenz steht und die betreffende Handlung nicht nach gesetzlichen Vorschriften erlaubt ist, ist für die oben aufgeführten Weiterverwendungen des Materials die Einwilligung des jeweiligen Rechteinhabers einzuholen.

Weitere Details zur Lizenz entnehmen Sie bitte der Lizenzinformation auf http://creativecommons.org/ licenses/by/4.0/deed.de.

\section{Chibber, Vivek: Postkoloniale Theorie und das Gespenst des Kapitals, 382 S., Dietz, Berlin 2018.}

\section{Sebastian Schlund}

Online publiziert: 30. April 2020

(C) Der/die Autor(en) 2020

Vivek Chibber tritt mit dem Anspruch an, fundamentale Kritik an zentralen Annahmen der Subaltern Studies als Teilbereich der postkolonialen Theorie zu üben, die er als „,diffuses und nebulöses Gedankengut“" empfindet (S. 354). Entsprechend scharf schießt der Soziologieprofessor der New York University auch und schreckt nicht vor absoluten Zurückweisungen solch renommierter Personen wie Dipesh Chakrabarty, Ranajit Guha und Partha Chaterjee zurück. Chibber nimmt seine Leserschaft

\footnotetext{
S. Schlund $(\bowtie)$

Christian-Albrechts-Universität zu Kiel, Kiel, Deutschland

E-Mail: s.schlund@collegiumphilosophicum.uni-kiel.de
} 